Pacific Journal of Mathematics

FUNCTIONAL REPRESENTATION OF TOPOLOGICAL
ALGEBRAS 


\title{
FUNCTIONAL REPRESENTATION OF TOPOLOGICAL ALGEBRAS
}

\author{
Peter D. Morris and Daniel E. Wulbert
}

A topological algebra $E$ is an algebra over the real or complex numbers together with a topology such that $E$ is a topological vector space and wach that multiplication in $E$ is jointly continuous. For a topological space $X, C(X)$ denotes the algebra of all continuous, complex-valued functions on $X$ with the usual pointwise operations. Unless otherwise stated, $C(X)$ is assumed to have the compact-open topology. Our principal concern is with representing (both topologically and algebraically) a commutative (complex) topological algebra, with identity, $E$ as a subalgebra of some $C(X), X$ a completely regular Hausdorff space. We obtain several characterizations of topological algebras which can be so represented. The most interesting of these is that the topology on $E$ be generated by a family of semi-norms each of which behaves, with respect to the multiplication in the algebra, like the norm in a (Banach) function algebra.

Let $M$ be the set of nonzero, continuous, multiplicative, linear functionals on a topological algebra $E$, provided with the weak topology induced by $E$. We are especially interested in representing $E$ as a subalgebra of $C(M)$. Our results along this line are found in $\S 4$. If $E$ is also provided with an involution, we wish to represent $E$ in such a way that involution goes over into complex conjugation. This problem is studied in $\S 5$.

The principal known results along the lines of our investigation are due to Arens and Michael. Arens ([3], Th. 11.4) characterized the topological algebras which are topologically isomorphic to $C(X)$, for $X$ a paracompact space. Michael ([9], Th. 8.4, p. 33) obtained sufficient conditions for a topological algebra to be topologically isomorphic to $\left(C(M), T_{0}\right)$, where $T_{0}$ is a topology weaker than the compactopen topology. Arens ([3], Th. 11.6) obtained a similar result. We obtain Michael's result as Corollary 5.3.

In $\S 2$ we prove that a Hausdorff space $\mathrm{X}$ is completely regular if and only if the closed ideals in $C(X)$ are in one-to-one correspondence (in the usual way) with the closed subsets of $X$. The necessity is well known in case $X$ is compact but our theorem seems to be new. In $\S 3$ we characterize those spaces $C(X)$ which have the Mackey topology. This section is unrelated to the rest of the paper but is of some interest in itself.

In $\S 6$ we apply some of our previous results to the general study 
of topological algebras. Section 7 is concerned with a concept, that of a countably barrelled topological vector space, recently introduced by Taqdir Husain [7]. We characterize those space $C(X)$ which are countably barrelled and then give examples to show that not every $C(X)$ is countably barrelled and to show that there exist countably barrelled nonbarrelled spaces.

We take the opportunity to explain some of our notation and terminology. If $E$ is a topological vector space, then $E^{\prime}$ denotes the topological dual of $E$.

The concept of a locally $m$-convex topological algebra was introduced by Arens [1]. Let $E$ be a topological algebra. Then $E$ is locally $m$-convex if, and only if, $E$ admits a basis for the neighborhoods of 0 consisting of balanced, convex, closed sets $U$ such that $U^{2}=U U \subset U$. Equivalently, $E$ is locally $m$-convex if, and only if, there is a set $\left\{p_{i}: i \in I\right\}$ of semi-norms on $E$ such that: (i) the sets of the form $\left\{x \in E: p_{i}(x) \leqq b\right\}$, for $i \in I, b>0$, form a base of neighborhoods of 0 ; and (ii)

$$
p_{i}(x y) \leqq p_{i}(x) p_{i}(y),
$$

for $i \in I, x, y \in E$. The standard work on locally $m$-convex algebras is the Memoir of Michael [9].

If $X$ is a topological space, then $C(X)$ is locally $m$-convex. Simply take the semi-norms $\left\{p_{K}: K\right.$ a compact subset of $\left.X\right\}$ defined by $p_{K}(f)=\sup \{|f(x)|: x \in K\}$, for $f \in C(X)$. The same is true of any subalgebra of $C(X)$.

Throughout this paper, all topological algebras are assumed to have identities and be commutative. As topological vector spaces, they are assumed to be locally convex and Hausdorff. The scalar field is always the complex numbers.

2. Closed ideals in $C(X)$. Let $X$ be a Hausdorff space. If $A$ is a closed subset of $X$, let $I_{A}=\{f \in C(X): f$ vanishes on $A\}$. Then $I_{A}$ is a closed ideal in $C(X)$. It is well-known that all closed ideals are of this form if $X$ is compact and that, in fact, there is a one-toone correspondence between the closed ideals of $C(X)$ and the closed subsets $A$ of $X$ via the relation $A \leftrightarrow I_{A}$. We have the following characterization of the spaces for which this result holds:

Theorem 2.1. The closed ideals in $C(X)$ are in one-to-one correspondence with the closed subsets of $X$ via $A \leftrightarrow I_{A}$ if, and only if, $X$ is completely regular.

Proof. Suppose $X$ is completely regular. Let $I$ be a closed ideal in $C(X)$. Let $A=\{x \in X: f(x)=0$ for all $f \in I\}$. Then $A$ is a closed (possibly empty) set. We show that $I=I_{A}$. Clearly $I \subseteq I_{A}$. Let $K$ 
be a compact subset of $X$, and let $I^{\prime}$ and $I_{A}^{\prime}$ denote the collections of functions in $C(K)$ which are restrictions to $K$ of functions in $I$ and $I_{A}$, respectively. Now $I^{\prime}$ and $I_{A}^{\prime}$ are ideals in $C(K)$. Since $K$ is compact, the closure (in $C(K)$, of $I^{\prime}$ is the ideal in $C(K)$ of functions vanishing on $K \cap A$. In particular, $I_{A}^{\prime} \subseteq C l I^{\prime}$. Hence $I$ is dense in $I_{A}$ and, since $I$ and $I_{A}$ are closed, $I=I_{A}$. By the complete regularity of $X, I_{A} \neq I_{B}$ if $A \neq B$.

If $X$ is not completely regular, then there exists a closed subset $A$ of $X$ and a point $x \in X \backslash A$ such that every function in $C(X)$ that vanishes on $A$ also vanishes at $x$. Letting $B=A \cup\{x\}$, we have $I_{A}=I_{B}$ with $A \neq B$. This completes the proof.

CoRollary 2.2. Let $X$ be completely regular. The closed maximal ideals in $C(X)$ are in one-to-one correspondence with the points of $X$ via the relation $x \leftrightarrow I_{\{x\}}$.

CoRollary 2.3. Let $X$ be completely regular. The nonzero, continuous, multiplicative, linear functions on $C(X)$ (i.e. the continuous algebraic homomorphisms from $C(X)$ into the complex numbers) are in one-to-one correspondence with the points of $X$ via the relation $x \leftrightarrow m_{x}$, where $m_{x}(f)=f(x)$, for $f \in C(X)$.

3. The Mackey topology on $C(X)$. If $E$ is a locally convex Hausdorff topological vector space with dual space $E^{\prime}$, then the Mackey topology on $E$ is the topology of uniform convergence on the weakly compact, convex, balanced subsets of $E^{\prime}$ (see [8], p. 173).

If $X$ is a completely regular Hausdorff space and if $L$ is continuous linear function on $C(X)$, then the support of $L$, $\operatorname{supp}(L)$, is the smallest closed set $A$ such that $L(f)=0$ for every $f \in C(X)$ vanishing on $A$. It is not difficult to show that $\operatorname{supp}(L)$ always exists and is compact. If $B \subseteq C(X)^{\prime}$, then the support, $\operatorname{supp}(B)$, of $B$ is defined to be $\operatorname{cl}(\cup\{\operatorname{supp}(L): L \in B\})$.

Lemma 3.1. Let $X$ be a completely regular Hausdorff space. A subset $B$ of $C(X)^{\prime}$ is equicontinuous if, and only if, the support $K$ of $B$ is compact and

$$
\sup \left\{|L(f)|: L \in B, f \in C(X), p_{k}(f) \leqq 1\right\}<\infty .
$$

Proof. Necessity. Since $B$ is equicontinuous it is contained in the polar of some neighborhood of 0 in $C(X)$. That is, for some $n>0$ and some compact $H \subseteq X$,

$B \subseteq n\left\{L \in C(X)^{\prime}:|L(f)| \leqq 1\right.$ for every $f \in C(X)$ with $\left.p_{H}(f) \leqq 1\right\}$. Let $L \in B$ and suppose $f \in C(X)$ and $f$ vanishes on $H$. Then 


$$
p_{H}(m f)=0 \leqq 1,
$$

for all $m>0$. Hence $m|L(f)| \leqq 1$ for all $m>0$. Therefore $L(f)=0$. We conclude that $\operatorname{supp}(L) \subseteq H$ and hence that $\operatorname{supp}(B) \subseteq H$ and is therefore compact. Let $K=\operatorname{supp}(B)$ and let $f \in C(X)$ be such that $p_{k}(f)=1$. There exists an extension $f^{\prime} \in C(X)$ of the restriction of $f$ to $K$ such that $p_{H}\left(f^{\prime}\right) \leqq 1$. Then $\left|L\left(f^{\prime}\right)\right| \leqq n$, for all $L \in B$. On the other hand, $f-f^{\prime}$ vanishes on $K$ so that $L(f)=L\left(f^{\prime}\right)$ for all $L \in B$. Hence

$$
\sup \left\{|L(f)|: L \in B, f \in C(X), p_{k}(f) \leqq 1\right\} \leqq n,
$$

and the necessity is proved.

Sufficiency. Let

$$
r=\sup \left\{|L(f)|: L \in B, f \in C(X), p_{k}(f) \leqq 1\right\} .
$$

We clearly have

$$
B \subseteq r\left\{L \in C(X)^{\prime}:|L(f)| \leqq 1 \text { for all } f \in C(X) \text { with } p_{k}(f) \leqq 1\right\} .
$$

Hence $B$ is contained in the polar of a neighborhood of 0 in $C(X)$ and is therefore equicontinuous.

This completes the proof.

THEOREM 3.2. Let $X$ be a completely regular Hausdorff space. The following statements are equivalent:

(i) $C(X)$ has the Mackey topology;

(ii) each weakly compact, balanced, convex subset of $C(X)^{\prime}$ is contained in a multiple of the bipolar of some weakly compact set of continuous multiplicative linear functionals;

(iii) if $B$ is a weakly compact, convex, balanced subset of $C(X)^{\prime}$, then $\operatorname{supp}(B)$ is compact.

Proof. $C(X)$ has the Mackey topology if, and only if every weakly compact, balanced, convex subset of $C(X)^{\prime}$ is equicontinuous. From the fact (Cor. 2.3) that continuous, multiplicative, linear functionals correspond to points of $X$, it is clear that the multiples of bipolars of weakly compact sets of continuous, multiplicative linear functionals are precisely the polars of basic 0-neighborhoods in $C(X)$. Since a subset of $C(X)^{\prime}$ is equicontinuous if, and only if, it is contained in the polar of some 0 -neighborhood in $C(X)$, the proof that (i) $\Leftrightarrow$ (ii) follows. From (3.1), the fact that (i) $\Rightarrow$ (iii) is clear. To show that (iii) $\Rightarrow$ (i) let $B$ be a weakly compact balanced, convex subset of $C(X)^{\prime}$. By (iii) the support $K$ of $B$ is compact. Since $B$ is weakly compact, the set $\{|L(f)|: L \in B\}$ is bounded for each $f \in C(X)$. For each $L \in B$, we 
define a functional $L^{*}$ on $C(K)$ by: $L^{*}(h)=L\left(h^{\prime}\right)$, where $h \in C(K)$ and $h^{\prime}$ is any continuous extension of $h$ to all of $X$. This definition makes sense because, if $h^{\prime \prime}$ is a second continuous extension of $h$ to $X$, then $h^{\prime \prime}-h^{\prime}$ vanishes on $K$ so that $L\left(h^{\prime \prime}\right)=L\left(h^{\prime}\right)$. To show that $L^{*} \in C(K)^{\prime}$, it suffices to show that $L$ is bounded on $\left\{f \in C(X): p_{k}(f) \leqq 1\right\}$ since the compact-open topology on $C(K)$ is the same as the supremum norm topology. This, however, follows from the continuity of $L$ and the fact that $\operatorname{supp}(L) \subseteq K$. Let $B^{*}=\left\{L^{*}: L \in B\right\}$. From the definition of $L^{*}$, we have that $\left\{\left|L^{*}(h)\right|: L^{*} \in B^{*}\right\}$ is bounded for each $h \in C(K)$. By the Uniform Boundedness Principle, $B^{*}$ is bounded in norm. In other words, by the definition of $L^{*}$ and $B^{*}$,

$$
\sup \left\{|L(f)|: L \in B, f \in C(X), p_{k}(f) \leqq 1\right\}<\infty .
$$

Hence, by (3.1), $B$ is equicontinuous. Therefore $C(X)$ has the Mackey topology.

This completes the proof.

Many spaces $C(X)$ do have the Mackey topology. For example, letting $R$ denote the real numbers, $C(R)$ is barrelled and therefore has the Mackey topology (see [10] or [12]). The theorem has some point, however, since not every $C(X)$ has the Mackey topology. Let $W$ be the space of ordinals less than the first uncountable ordinal. Then $C(W)$ does not have the Mackey topology. We sketch a proof of this fact. For $\sigma \in W$, let $m_{\sigma} \in C(W)^{\prime}$ be defined by $m_{\sigma}(f)=f(\sigma)$, for $f \in C(W)$. Let $A=\left\{m_{\sigma+1}-m_{\sigma}: \sigma \in W\right\}$ and let $B$ be the closed, balanced, convex hull of $A$. It is easy to see that $A$ is not equicontinuous. To complete the proof, one then shows that $B$ is weakly compact. This example was inspired by a similar one in [4].

Since a barrelled space has the Mackey topology, we see that if $C(X)$ is barrelled, then every weakly compact, convex, balanced subset of $C(X)^{\prime}$ has compact support. As an impovement on this result we have the following proposition. The proof is a slight variation of the proof of a result of Nachbin's ([10], Th. 1) and is therefore omitted.

Let $A$ be a subset of a topological space $X$. Then $A$ is said to be $C(X)$-pseudocompact if, and only if, every function in $C(X)$ is bounded on $A$. Note that a $C(X)$-pseudocompact set need not be a pseudocompact space.

Proposition 3.3. Let $X$ be a completely regular Hausdorff space. Then the support of every weakly bounded (and hence every weakly compact) subset of $C(X)^{\prime}$ is $C(X)$-pseudocompact. In addition, $C(X)$ is barrelled if and only if the support of each such set is compact.

4. Functional representations of topological algebras. Let $E$ 
be a topological algebra and let $M$ be the set of nonzero, continuous, multiplicative, linear functionals on $E$. Call $M$ the carrier of $E$. Conceivably, $M$ may be empty. If it is not, we topologize it as follows. Let $E^{\prime}$ have the weak topology and let $M$ have the relative topology it inherits as a subset of $E^{\prime}$. With this topology, $M$ is a completely regular Hausdorff space. We construct a mapping $G: E \rightarrow C(M)$ by defining $G(x)(m)=m(x)$ for each $x \in E$ and $m \in M$. Call $G$ the Gelfand map of $E$. Clearly $G$ is an algebraic homomorphism.

We turn to the question of when $G$ is an isomorphism. Let us call $E$ strongly semi-simple if $x \in E, x \neq 0$ imply $m(x) \neq 0$ for some $m \in M$. It is then clear that $G$ is an algebraic isomorphism of $E$ into $C(M)$ if, and only if, $E$ is strongly semi-simple.

A few remarks concerning this definition of strong semi-simplicity are in order. First, if $X$ is a completely regular space and if $E$ is a subalgebra of $C(X), E$ is strongly semi-simple. This follows from (2.3). Secondly, if $E$ is a topological algebra with nonvoid carrier $M$, we may, by passing to a quotient algebra $F$ of $E$, obtain a strongly semi-simple algebra with the same carrier $M$. Simply let

$$
I=\{x \in E: m(x)=0
$$

for all $m \in M\}$ and let $F=E / I$. Finally, under suitable restrictions on $E$, strong semi-simplicity is equivalent to the property that the intersection of the closed maximal ideals of $E$ be $\{0\}$, i.e., semi-simplicity. That this is the case for locally $m$-convex algebra follows from ([9], Prop. 2.4e and Prop. 2.8) and Arens' generalization [2] of the Gelfand-Mazur Theorem.

We turn now to the question of the continuity of $G$ and, if defined, its inverse.

THEOREM 4.1. Let $E$ be a topological algebra with nonvoid carrier $M$ and Gelfand map $G$. If $E$ is barrelled, then $G$ is continuous.

Proof. Let $x_{i}, i \in I$, be a net in $E$ converging to $x \in E$. Since $E$ is barrelled, $x_{i}$ converges to $x$, uniformly on compact subsets of $E^{\prime}$ (regarding $E$ as a set of functions on $E^{\prime}$ ). Hence $G\left(x_{i}\right)$ converges to $G(x)$, uniformly on compact subsets of $M$. Therefore $G$ is continuous.

It is easy to show that $G$ continuous does not imply $E$ barrelled. Let $R$ be the real numbers and let $C^{*}(R)$ be the bounded functions in $C(R)$. Then $C^{*}(R)$ is not barrelled (since $\left\{f \in C^{*}(R):|f(r)| \leqq 1, r \in R\right\}$ is a barrel but not a 0 -neighborhood) but $G$ is continuous.

THEOREM 4.2. Let $E$ be a strongly semi-simple topological algebra with carrier $M$ and Gelfand map $G$. If every equicontinuous subset of $E^{\prime}$ is contained in a multiple of the closed, convex, balanced hull 
of some compact subset of $M$, then $G^{-1}$ is continuous.

Proof. The polars of equicontinuous subsets of $E^{\prime}$ form a base of 0 -neighborhoods in $E$. Let $A \subseteq E^{\prime}$ be equicontinuous. By hypothesis, there is a compact subset $K$ of $M$ and $n>0$ such that $n K^{00} \supseteqq A$. Taking polars on both sides, we have $1 / n K^{0} \cong A^{0}$. Hence

$$
1 / n G\left(K^{0}\right) \subseteq G\left(A^{0}\right) \text {. }
$$

Now let

$$
W=\{f \in C(M):|f(m)| \leqq 1, m \in K\} .
$$

Then $W$ is a 0 -neighborhood in $C(M)$. We have

$$
\frac{1}{n}(W \cap G(E))=\frac{1}{n} G\left(K^{0}\right) \subseteq G\left(A^{0}\right) .
$$

This shows that $G$ is open and hence that $G^{-1}$ is continuous.

COROLlary 4.3. Let $E$ be a strongly semi-simple topological algebra with carrier $M$ and Gelfand map $G$. If the multiples of the polars of equicontinuous compact subsets of $M$ form a base of 0-neighborhoods in $E$, then $G^{-1}$ is continuous.

We need the following:

Definition 4.4. Let $E$ be a topological algebra. Then $E$ is pseudo-normed if, and only if, there is a set $\left\{p_{i}: i \in I\right\}$ of semi-norms on $E$ such that: (i) the collection of sets $\left\{x \in E: p_{i}(x) \leqq b\right\}$, for $i \in I$, $b>0$, form a neighborhood base at 0 ; (ii) $p_{i}(x y) \leqq p_{i}(x) p_{i}(y)$, for $i \in I$, $x, y \in E$; (iii) $p_{i}\left(x^{2}\right)=p_{i}(x)^{2}, i \in I, x \in E$.

Note that a pseudo-normed algebra is locally $m$-convex. Also, if $X$ is a topological space, then, with the semi-norms $\left\{p_{K}: K\right.$ a compact subset of $X$ \}, any subalgebra of $C(X)$ is pseudo-normed.

If $M$ is the nonvoid carrier of the topological algebra $E$, let $T_{0}$ denote the topology on $C(M)$ of uniform convergence on closed equicontinuous subsets of $M$. Each such set is compact and therefore $T_{0}$ is weaker than the compact-open topology. The topology $T_{0}$ was first defined by Michael ([9], p. 32).

THEOREM 4.5. Let E be a strongly semi-simple topological algebra with carrier $M$ and Gelfand map $G$. The following are equivalent:

(i ) $E$ is pseudo-normed;

(ii) $E$ can be embedded algebraically and topologically in a 
product algebra $\prod_{i \in I} C\left(K_{i}\right)$, where $K_{i}$ is a compact Hausdorff space for each $i \in I$;

(iii) $E$ can be embedded algebraically and topologically in a $C(X)$ for $X$ a locally compact Hausdorff space;

(iv) $E$ can be embedded algebraically and topologically in a $C(X)$ for $X$ a completely regular Hausdorff space;

( v ) $G^{-1}:\left(C(M), T_{0}\right) \rightarrow E$ is continuous;

(vi) $G: E \rightarrow\left(C(M), T_{0}\right)$ is a topological and algebraic isomorphism (into).

Proof. (i) $\Rightarrow$ (ii): Let $\left\{p_{i}: i \in I\right\}$ be as in the definition of a pseudo-normed algebra. For each $i \in I$, let $N_{i}=\left\{x \in E: p_{i}(x)=0\right\}$. Then $N_{i}$ is a closed ideal. Let $A_{i}=E / N_{i}$, equipped with the norm $q_{i}$, where $q_{i}\left(x+N_{i}\right)=p_{i}(x)$, for $x \in E$. Let $C_{i}$ be the completion of $A_{i}$. Then $C_{i}$ is a Banach algebra and $q_{i}\left(\hat{x}^{2}\right)=q_{i}(\hat{x})^{2}$, for $\widehat{x} \in C_{i}$. Thus $C_{i}$ is semisimple and moreover the Gelfand map carrying $C_{i}$ into $C\left(K_{i}\right)$, where $K_{i}$ is the (compact) carrier of $C_{i}$, is an isometric isomorphism (see [11], p. 194, IV). Now according to ([9], Prop. 2.7, p. 10), since $E$ is locally $m$-convex, $E$ is topologically and algebraically isomorphic to a subalgebra of $\Pi\left\{A_{i}: i \in I\right\}$ and therefore to a subalgebra of $\Pi\left\{C_{i}: i \in I\right\}$ and therefore to a subalgebra of $\Pi\left\{C\left(K_{i}\right): i \in I\right\}$. This proves (ii).

(ii) $\Rightarrow$ (iii): Let $K$ be the free union (see [5], p. 132) of the $K_{i}, i \in I$. It is clear that $C(K)$ is topologically isomorphic to

$$
\Pi\left\{C\left(K_{i}\right): i \in I\right\} \text {. }
$$

Since $K$ is locally compact, (iii) is proved.

(iii) $\Longrightarrow$ (iv): Obvious.

(iv) $\Rightarrow(\mathrm{v})$ : Without loss of generality, we can assume that $E$ contains the constant functions in $C(X)$. Now each point of $X$ determines an element of $M$, although two distinct points of $X$ may determine the same element of $M$. The mapping $h$ of $X$ into $M$ thus determined, is continuous. Let $U=\{f \in E:|f(x)| \leqq \varepsilon, x \in K\}$ be a basic 0 -neighborhood in $E$, where $K \subseteq X$ is compact and $\varepsilon>0$. Then $h(K)$ is a compact subset of $M$ and

$\varepsilon h(K)^{0}=\{f \in E:|m(f)| \leqq \varepsilon, m \in h(K)\}=\{f \in E:|f(x)| \leqq \varepsilon, x \in K\}=U$.

This shows that the multiples of polars of sets of the form $h(K)$ form a base of 0 -neighborhoods in $E$. Then, as in (4.3), it follows that $G^{-1}:\left(C(M), T_{0}\right) \rightarrow E$ is continuous.

$(\mathrm{v}) \Rightarrow(\mathrm{vi})$ : We already know that $G$ is an algebraic isomorphism so it remains to show that $G$ is continuous. Let $K$ be a closed equicontinuous subset of $M$ and let $U=\{G(f) \in G(E):|G(f)(x)| \leqq 1, x \in K\}$. Then $G^{-1}(U)=\{f \in E:|m(f)| \leqq 1\}=K^{0}$. But polars of equicontinuous 
subsets of the dual of a locally convex space are always 0-neighborhoods. This proves (vi).

(vi) $\Rightarrow\left(\right.$ i ): For each closed equicontinuous $K \cong M$, let $q_{K}(f)=$ sup $\{|G(f)(x)|: x \in K\}, f \in E$. Then clearly $\left\{q_{K}: K \subseteq X\right.$ closed and equicontinuous satisfies the conditions in the definition of a pseudo-normed algebra. This completes the proof.

Let $E, M, G$ be as above. If $G$ is continuous (e.g., if $E$ is barrelled), we get the following stronger result. The proof is omitted.

THEOREM 4.6. Let $E$ be a strongly semi-simple topological algebra with carrier $M$ and Gelfand map $G$. If $G$ is continuous, the following statements are equivalent:

(i) $E$ is pseudo-normed;

(ii) $E$ can be embedded algebraically and topologically in an algebra $\prod_{i \in I} C\left(K_{i}\right)$, where $K_{i}$ is a compact Hausdorff space for each $i \in I$;

(iii) $E$ can be embedded algebraically and topologically in a $C(X)$ for $X$ a locally compact Hausdorff space;

(iv) $E$ can be embedded algebraically and topologically in a $C(X)$ for $X$ a completely regular Hausdorff space;

(v) $G$ is a homeomorphism.

5. Topological algebras with involution. We need the following:

Definition 5.1. A topological algebra $E$ is called an algebra with involution if there is a mapping $x \rightarrow x^{*}$ of $E$ onto itself satisfying, for $x, y \in E$ and $\beta$ a scalar,

(i) $x^{* *}=x$;

(ii) $(x+y)^{*}=x^{*}+y^{*}$

(iii) $(\beta x)^{*}=\bar{\beta} x^{*}(\bar{\beta}=$ complex conjugate);

(iv) $(x y)^{*}=x^{*} y^{*}$.

$E$ is a *algebra if it is an algebra with involution admitting a set $\left\{p_{i}: i \in I\right\}$ of semi-norms satisfying

(i ) $p_{i}(x y) \leqq p_{i}(x) p_{i}(y)$, for $x, y \in E$;

(ii)' the sets $\left\{x \in E ; p_{i}(x)<b\right\}$, for $i \in I, b>0$, form a base of 0 -neighborhoods in $E$;

(iii) $p_{i}\left(x x^{*}\right)=p_{i}(x)^{2}$, for $x \in E$.

Michael ([9], Dfn. 6.3, p. 23) calls our algebras with involution, *-algebras. A *-algebra is locally $m$-convex and, in fact, pseudonormed. The proof of this fact is omitted (see [11], p. 230). For a topological space $X$, the usual involution on $C(X)$ is complex conjuga- 
tion. If $E$ is a subalgebra of a $C(X)$ such that $f \in E$ implies $\bar{f} \in E$, then $E$ is said to be self-adjoint. Obviously, a self-adjoint subalgebra of a $C(X)$ is a *-algebra with the usual semi-norms. If $E$ and $F$ are algebras with involution, then $H: E \rightarrow F$ is a ${ }^{*}$-homomorphism if $H$ is an algebraic homomorphism and $H\left(x^{*}\right)=H(x)^{*}$, for $x \in E$.

We characterize ${ }^{*}$-algebras in the following;

THEOREM 5.2. Let $E$ be a topological *-algebra with carrier $M$ and Gelfand map $G$. Then $E$ is a strongly semi-simple *-algebra if, and only if $, G: E \rightarrow\left(C(M), T_{0}\right)$ is a topological *-isomorphism onto $a$ dense (in the compact-open topology) self-adjoint subalgebra of $C(M)$.

Proof. Sufficiency is clear. If $E$ is a strongly semi-simple *-algebra, then $G: E \rightarrow\left(C(M), T_{0}\right)$ is already known to be a topological and algebraic isomorphism. To complete the proof, it suffices to show that $G$ is a *-isomorphism. That this will imply that $G$ is self-adjoint is clear. The fact that $G(E)$ is dense then follows from the StoneWeierstrass Theorem generalized to arbitrary $C(X)$ (see [5], p. 283, or [9], Prop. 6.8, p. 24). To shows that $G$ is a *-isomorphism, let $m_{0} \in M$. Since $\left\{m_{0}\right\}$ is compact, there is $i \in I$ and $b \in(0,1)$ such that

$$
D=\left\{x \in E: p_{i}(x)<b\right\} \subseteq\left\{x \in E:\left|m_{0}(x)\right|<1\right\} \text {. }
$$

Let $K=D^{0} \cap M$. Then $K$ is compact. Let $N_{i}=\left\{x \in E: p_{i}(x)=0\right\}$ and let $A_{i}=E / N_{i}$ provided with the norm $q_{i}$ defined by

$$
q_{i}\left(x+N_{i}\right)=p_{i}(x),
$$

for $x \in E$. Finally, let $C_{i}$ be the completion of $A_{i}$. Now if $x \in E$, then it is easily seen that $p_{i}(x)=p_{i}\left(x^{*}\right)$. Hence $x, y \in E, x-y \in N_{i}$ imply $x^{*}-y^{*} \in N_{i}$. Therefore the formula $\left(x+N_{i}\right)^{*}=x^{*}+N_{i}$ defines an involution on $A_{i}$. Moreover $q_{i}\left(x x^{*}\right)=q(x)^{2}$ for $x \in A_{i}$. Hence the involution may be extended to $C_{i}$ so that $C_{i}$ is a $B^{*}$-algebra. By a well known theorem (see [11], p. 230, Th. 1), $C_{i}$ is topologically *-isomorphic to $C\left(K_{i}\right)$, where $K_{i}$ is its carrier. Let $G_{i}$ be the Gelfand map of $C_{i}$. It is routine to verify that there is a homomorphism $m \rightarrow m_{i}$ of $K$ onto $K_{i}$ and that, for $m \in K$ and $x \in E$,

$$
G_{i}\left(x+N_{i}\right)\left(m_{i}\right)=G(x)(m) .
$$

Now it is well known that $G_{i}$ is a *-isomorphism so that, if $x \in E$, $G_{i}\left(x^{*}+N_{i}\right)\left(m_{i}\right)$ is the complex conjugate of $G_{i}\left(x+N_{i}\right)\left(m_{i}\right)$. Hence $G\left(x^{*}\right)(m)$ is the complex conjugate of $G(x)(m)$. In particular, this is true for $m=m_{0}$. Since $m_{0}$ is arbitrary, $G$ is a ${ }^{*}$-isomorphism. This completes the proof. 
It should be noted that a *-algebra is automatically strongly semisimple. To prove this, let $E$ be a *algebra and let $x$ be a nonzero element of $E$. Then there is a semi-norm $p_{i}$ such that $p_{i}(x) \neq 0$. Letting $N_{i}, A_{i}, p_{i}$, and $C_{i}$ be as in the previous proof, we have that $x+N_{i}$ is a nonzero element of $C_{i}$. Since $C_{i}$ is semi-simple, there is a continuous multiplicative linear functional $m_{i}$ on $C_{i}$ such that

$$
m_{i}\left(x+N_{i}\right) \neq 0 \text {. }
$$

Let $m$ be the continuous multiplicative linear functional on $E$ corresponding to $m_{i}$ as in the previous proof. Then $m(x) \neq 0$. Hence $E$ is strongly semi-simple.

As a corollary to Theorem (5.2), we get a result due to Michael ([9], Th. 8.4, P. 33).

Corollary 5.3. Let $E$ be a complete *-algebra. Then $E$ is topologically *-isomorphic to $\left(C(M), T_{0}\right)$.

We remark that other conditions equivalent to $E$ being a *algebra are now easily derived using (4.5). For example, $E$ is a *-algebra if, and only if, $E$ is topologically *-isomorphic to a self-adjoint subalgebra of $C(X)$, for some completely regular Hausdorff space $X$.

As in $\S 4$, if $G$ is known to be continuous (e.g., if $E$ is barrelled), we can replace $T_{0}$ with the compact-open topology in (5.2) and (5.3).

Recall that a completely regular space $X$ is realcompact if, and only if, the only multiplicative linear functions on $C(X)$ are the evaluations at points of $X$ (see [6]). According to a result of Nachbin [10] and Shirota [12], $C(X)$, for $X$ completely regular Hausdorff, is bornological if, and only if, $X$ is realcompact. From the fact that a complete bornological space is barrelled, we have:

Corollary 5.4. A complete topological *algebra $E$ is topologically *-isomorphic to $C(X)$, for some realcompact $X$ if, and only if, $E$ is bornological.

The following result is an easy consequence of (5.2). For a theorem of a similar type, see [13], Th. 3, p. 268.

CoRollary 5.5. Let $X$ be a realcompact space and $T$ a topology on $C(X)$ such that $(C(X), T)$ is a complete topological algebra. Then $T$ is the compact-open topology if, and only if, $(C(X), T)$ is a bornological *-algebra in which all the evaluations at points of $X$ are continuous.

The final result of this section implies a weak version of a theorem 
of Michael ([9], Th. 12.6, p. 53)

COROLLARY 5.6. Each multiplicative linear functional on a complete bornological *-algebra is continuous.

\section{Applications to topological algebras.}

THEOREM 6.1. Let $E$ be a barrelled topological algebra with nonvoid carrier $M$. If $A$ is a closed subset of $M$ then $A$ is compact if, and only if $\{m(x): m \in A\}$ is bounded for every $x \in E$.

Proof. Necessity is clear. If $\{m(x): m \in A\}$ is bounded for every $x \in E$, then $A$ is weakly bounded. But in the dual of a barrelled space, a set is weakly compact if, and only if, it is closed and weakly bounded ([8], Th. 18.7, (iii), p. 171). The proof follows from the observation that $M$ is weakly closed in $E^{\prime}$.

CoRollary 6.2. Let $E$ be a barrelled topological algebra with nonvoid carrier $M$. Then $M$ is compact if, and only if, the image of $E$ under the Gelfand map is contained in the bounded functions in $C(M)$.

COROLLARY 6.3. Let $E$ be a barrelled topological algebra with nonvoid carrier $M$. Then $C(M)$ is barrelled.

Proof. Note that, by (6.1), a closed $C(M)$-pseudocompact subset of $M$ is compact. According to a well known result due independently to Nachbin [10] and Shirota [12], the real space $C(X, R)$ is barrelled if, and only if, every $C(X, R)$-pseudocompact subset of $X$ is compact. Omitting the routine proof that $C(X, R)$ is barrelled if, and only if, $C(X)$ is, the proof is complete.

We are now in a position to give a simple proof of a theorem due to Warner ([13], Th. 4, p. 269). Call a topological algebra $E$ with nonvoid carrier $M$ full if the Gelfand map is an algebraic isomorphism of $E$ onto $C(M)$ (see [9], Dfn. 8.3). A topological space $X$ is hemicompact if $X=\bigcup_{i=1}^{\infty} A_{i}$, where each $A_{i}$ is compact, and each compact subset of $X$ is contained in some $A_{i}$. Also, $X$ is a $k$-space provided a subset of $X$ is closed if its intersection with each compact subset of $X$ is closed.

Theorem 6.4. (Warner). Let $E$ be a full Frechet algebra with carrier $M$. Then the Gelfand map is a homeomorphism and $M$ is a hemicompact k-space. 
Proof. A Frechet space is barrelled and therefore the Gelfand map is continuous. By $(6.3), C(M)$ is barrelled. Therefore the closure of the Gelfand image of an open set has nonvoid interior. From this and the fact that $E$ is a Baire space, we see that $C(M)$ is a Baire space. By the Open Mapping Theorem ([8], Th. 11.4, P. 99), the Gelfand mapping is a homeomorphism. Since $C(M)$ is thus a Frechet space, $M$ is a hemicompact $k$-space, by a result of Warner ([13], Th. 2, p. 267). This completes the proof.

DEFINITION 6.5. Let $E$ be a topological algebra with nonvoid carrier $M$. If $x \in E$ and $\{m(x): m \in M\}$ is bounded, then $x$ is said to be bounded. If every $x \in E$ is bounded, $E$ is bounded.

DeFinition 6.6. Let $E$ be a barrelled semi-simple topological algebra, with carrier $M$. Let $G$ be the Gelfand Map. Denote by $T$ the relative topology (from $C(M)$ ) on $G(E)$ transferred to $E$. Let $E^{\#}$ be the dual of $(E, T)$.

It is clear that $T$ is weaker than the given topology on $E$, so that $E^{\#} \subseteq E^{\prime}$.

THEOREM 6.7. Let $E$ be a barrelled, semi-simple, topological algebra, with nonvoid carrier $M$. Then $E$ is normable if, and only if, $E$ is bounded and $E^{\#}=E^{\prime}$.

Proof. If $E$ is normable, then it is pseudo-normed and therefore the Gelfand map is a homeomorphism. Clearly $M$ is compact. The conclusion follows immediately. Suppose $E$ is bounded and $E^{*}=E^{\prime}$. By (6.1), $M$ is compact and therefore $C(M)$ and hence $G(E)$, is normable. Thus $G(E)$ is bornological and therefore has the Mackey topology. Since $E^{\sharp}=E^{\prime}$, both $T$ and the given topology on $E$ are compatible with duality $\left(E, E^{\prime}\right)$. Hence $T$ is identical with the given topology on $E$. Hence $G$ is a homeomorphism and $E$ is normable. This completes the proof.

7. Countably barrelled spaces. At the 1966 International Congress of Mathematicians, T. Husain introduced the concept of a countably barrelled space as a generalization of that of a barrelled space. (See also [7]). At the time, Professor Husain asked for examples of countably barrelled spaces which are not barrelled. With the methods used in this paper, we can present such an example.

Definition 7.1. A topological vector space $E$ is countably barrelled if every $\sigma\left(E^{\prime}, E\right)$-bounded subset of $E^{\prime}$ which is the countable union of equicontinuous sets is itself equicontinuous. 
THEOREM 7.2. Let $X$ be a completely regular Hausdorff space. Then $C(X)$ is countably barrelled if, and only if, every $C(X)$-pseudocompact subset of $X$ which is the closure of a countable union of compact sets is actually compact.

Proof. Necessity. Suppose $B$ is a $C(X)$-pseudocompact subset of $X$ and that $B=C l\left(\bigcup_{n=1}^{\infty} K_{n}\right)$, where each $K_{n}$ is a compact subset of $X$. For each $n=1,2, \cdots$, let $A_{n}=\left\{L \in C(X)^{\prime}: \operatorname{supp}(L) \subseteq K_{n}\right.$ and $|L(f)| \leqq 1$ for every $f \in C(X)$ with $\left.p_{K_{n}}(f) \leqq 1\right\}$. By (3.1), each $A_{n}$ is equicontinuous. Let $A=\bigcup_{n=1}^{\infty} A_{n}$. It is clear that $A$ is $\sigma\left(C(X)^{\prime}, C(X)\right)$ bounded since $\operatorname{supp}(A)=B$ is $C(X)$-pseudocompact. Hence if $C(X)$ is countably barrelled, $A$ is equicontinuous and therefore $B$ is compact by (3.1).

Sufficiency. Suppose $C(X)$ is not countably barrelled so that there exists a bounded $A=\bigcup_{n=1}^{\infty} A_{n}$, where each $A_{n}$ is an equicontinuous subset of $C(X)^{\prime}$, such that $A$ is not equicontinuous. By (3.1), supp $\left(A_{n}\right)$ is compact and, by (3.3), supp $(A)$ is $C(X)$-pseudocompact but, by (3.1), not compact. However it is clear $\operatorname{supp}(A)=C l\left(\bigcup_{n=1}^{\infty} \operatorname{supp}\left(A_{n}\right)\right)$. Hence $X$ contains a $C(X)$-pseudocompact subset which is the closure of a countable union of compact sets but is not compact.

This completes the proof.

Not every $C(X)$ is countably barrelled. Let $W^{*}$ denote the space of ordinals less than or equal to the first uncountable ordinal and let $T$ denote the Tychonoff plank. Then $T=C l\left(\bigcup_{n=1}^{\infty}\left(W^{*} \times\{n\}\right)\right)$. But $T$ is pseudocompact and each $W^{*} \times\{n\}$ is compact. Since $T$ is not compact, $C(T)$ is not countably barrelled.

For an example of a countably barrelled nonbarrelled space, let $W$ denote the space of ordinals less than the first uncountable ordinal. Since $W$ is pseudocompact, $C(W)$ is not barrelled. On the other hand, it is easy to see that the closure of a countable union of compact subsets of $W$ is compact. Hence $C(W)$ is countably barrelled.

\section{BiBLIOGRAPHY}

1. R. F. Arens, The space $L^{\omega}$ and convex topological rings, Bull. Amer. Math. Soc. 52 (1946), 931-935.

2. Linear topological division Algebras, Bull. Amer. Math. Soc. 53 (1947), 623-630.

3. - A generalization of normed rings, Pacific J. Math. 2 (1952), 455-471.

4. J. B. Conway, The strict topology in the space of measures, Bull. Amer. Math. Soc. 72 (1966), 75-78.

5. J. Dugundji, Topology, Allyn and Bacon, Inc., Boston, 1966.

6, L. Gillman and M. Jerison, Rings of Continuous Functions, Van Nostrand, Princeton, 1960 .

7. T. Husain, Two new classes of locally convex spaces, Math. Annalen 166 (1966), 289-299. 
8. J. L. Kelley, I. Namioka, et al., Linear Topological Spaces, Van Nostrand, Princeton, 1963.

9. E. A. Michael, Locally multiplicatively-convex topological algebras, Mem. Amer. Math. Soc. 11 (1951).

10. L. Nachbin, Topological vector spaces of continuous functions, Proc. Nat. Acad. Sci. U.S.A. 40 (1954), 471-474.

11. M. A. Naimark, Normed Rings, revised ed., P. Noordhoff Ltd., Groningen, The Netherlands, 1964.

12. T. Shirota, On locally convex vector spaces of continuous functions, Proc. Japan Acad. 30 (1954), 294-298.

13. S. Warner, The topology of compact convergence on continuous function spaces, Duke Math. J. 25 (1958), 265-282.

Received August 22, 1966. D. E. Wulbert was supported by a grant from the Air Force Office of Scientific Research, AF-AFOSR-1109-66, to the University of Texas.

Pennsylvania State University and

UNIVERSITY OF LUND

UNIVERSITY OF WASHINGTON 



\section{PACIFIC JOURNAL OF MATHEMATICS}

\section{EDITORS}

\section{H. SAMELSON}

Stanford University

Stanford, California

J. P. JANS

University of Washington

Seattle, Washington 98105

\section{J. DugundJI}

University of Southern California Los Angeles, California 90007

RICHARD ARENS

University of California

Los Angeles, California 90024

\section{ASSOCIATE EDITORS}

E. F. BECKENBACH
B. H. NeUmanN

\section{SUPPORTING INSTITUTIONS}

UNIVERSITY OF BRITISH COLUMBIA CALIFORNIA INSTITUTE OF TECHNOLOGY

UNIVERSITY OF CALIFORNIA

MONTANA STATE UNIVERSITY

UNIVERSITY OF NEVADA

NEW MEXICO STATE UNIVERSITY

OREGON STATE UNIVERSITY

UNIVERSITY OF OREGON

OSAKA UNIVERSITY

UNIVERSITY OF SOUTHERN CALIFORNIA

\author{
STANFORD UNIVERSITY \\ UNIVERSITY OF TOKYO \\ UNIVERSITY OF UTAH \\ WASHINGTON STATE UNIVERSITY \\ UNIVERSITY OF WASHINGTON \\ AMERICAN MATHEMATICAL SOCIETY \\ CHEVRON RESEARCH CORPORATION \\ TRW SYSTEMS \\ NAVAL ORDNANCE TEST STATION
}

Mathematical papers intended for publication in the Pacific Journal of Mathematics should be typewritten (double spaced). The first paragraph or two must be capable of being used separately as a synopsis of the entire paper. It should not contain references to the bibliography. Manuscripts may be sent to any one of the four editors. All other communications to the editors should be addressed to the managing editor, Richard Arens at the University of California, Los Angeles, California 90024.

50 reprints per author of each article are furnished free of charge; additional copies may be obtained at cost in multiples of 50 .

The Pacific Journal of Mathematics is published monthly. Effective with Volume 16 the price per volume ( 3 numbers) is $\$ 8.00$; single issues, $\$ 3.00$. Special price for current issues to individual faculty members of supporting institutions and to individual members of the American Mathematical Society: $\$ 4.00$ per volume; single issues $\$ 1.50$. Back numbers are available.

Subscriptions, orders for back numbers, and changes of address should be sent to Pacific Journal of Mathematics, 103 Highland Boulevard, Berkeley 8, California.

Printed at Kokusai Bunken Insatsusha (International Academic Printing Co., Ltd.), 7-17, Fujimi 2-chome, Chiyoda-ku, Tokyo, Japan.

\section{PUBLISHED BY PACIFIC JOURNAL OF MATHEMATICS, A NON-PROFIT CORPORATION}

The Supporting Institutions listed above contribute to the cost of publication of this Journal, but they are not owners or publishers and have no responsibility for its content or policies. 


\section{Pacific Journal of Mathematics}

\section{Vol. 22, No. $2 \quad$ February, 1967}

Paul Frank Baum, Local isomorphism of compact connected Lie groups ....

Lowell Wayne Beineke, Frank Harary and Michael David Plummer, On the

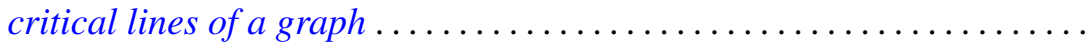

Larry Eugene Bobisud, On the behavior of the solution of the telegraphist's equation for large velocities .......................... 213

Richard Thomas Bumby, Irreducible integers in Galois extensions . . . . . . 221

Chong-Yun Chao, A nonimbedding theorem of nilpotent Lie algebras ..... 231

Peter Crawley, Abelian p-groups determined by their Ulm sequences ...... 235

Bernard Russel Gelbaum, Tensor products of group algebras ........... 241

Newton Seymour Hawley, Weierstrass points of plane domains .......... 251

Paul Daniel Hill, On quasi-isomorphic invariants of primary groups . . . . . 257

Melvyn Klein, Estimates for the transfinite diameter with applications to confomral mapping ................................ 267

Frederick M. Lister, Simplifying intersections of disks in Bing's side approximation theorem ............................. 281

Charles Wisson McArthur, On a theorem of Orlicz and Pettis ........... 297

Harry Wright McLaughlin and Frederic Thomas Metcalf, An inequality for

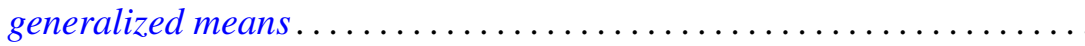

Daniel Russell McMillan, Jr., Some topological properties of piercing

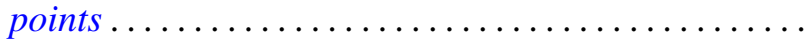

Peter Don Morris and Daniel Eliot Wulbert, Functional representation of topological algebras .

Roger Wolcott Richardson, Jr., On the rigidity of semi-direct products of Lie algebras..................................

Jack Segal and Edward Sandusky Thomas, Jr., Isomorphic

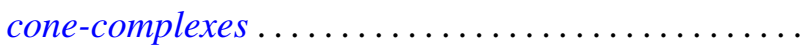

Richard R. Tucker, The $\delta^{2}$-process and related topics.... 